ARTICLE

https://doi.org/10.1038/s41467-019-08675-3

\title{
Amine-responsive cellulose-based ratiometric fluorescent materials for real-time and visual detection of shrimp and crab freshness
}

\author{
Ruonan Jia ${ }^{1,2}$, Weiguo Tian ${ }^{1}$, Haotian Bai ${ }^{3}$, Jinming Zhang (1) ${ }^{1}$, Shu Wang (D) $^{2,3}$ \& Jun Zhang (1) ${ }^{1,2}$
}

Herein, we design and prepare cellulose-based ratiometric fluorescent materials with superior amine-response, which offers the real-time and visual detection of seafood freshness. Through utilizing the reactive hydroxyl groups along cellulose chains, we covalently immobilize the fluorescein isothiocyanate (FITC) as indicator and protoporphyrin IX (PpIX) as internal reference onto cellulose acetate (CA), respectively. Subsequently, a series of dualemission solid fluorescent materials are achieved by simply blending green emitting CA-FITC with red-emitting CA-PpIX with varying ratios. They exhibit a sensitive, color-responsive, rapid and linear response to ammonia in a wide range of $5.0 \mathrm{ppm}$ to $2.5 \times 10^{4} \mathrm{ppm}$. Benefiting from the excellent solubility and processibility of cellulose derivatives, the as-prepared materials are readily processed into different material forms, including printing ink, coating, flexible film, and nanofibrous membrane. The electrospun nanofibrous membrane is successfully employed as a low-cost, high-contrasting, quick-responsive fluorescent trademark for visual monitoring the freshness of shrimp and crab.

\footnotetext{
${ }^{1}$ CAS Key Laboratory of Engineering Plastics and CAS Research/Education Center for Excellence in Molecular Sciences, Chinese Academy of Sciences, 100190 Beijing, China. ${ }^{2}$ College of Chemistry, University of Chinese Academy of Sciences, 100049 Beijing, China. ${ }^{3}$ CAS Key Laboratory of Organic Solids, Institute of Chemistry, Chinese Academy of Sciences, 100190 Beijing, China. Correspondence and requests for materials should be addressed to J.Z. (email: zhjm@iccas.ac.cn) or to S.W. (email: wangshu@iccas.ac.cn) or to J.Z. (email: jzhang@iccas.ac.cn)
} 
S imple, fast, low-cost and accurate monitoring systems for food safety are becoming more urgent and indispensable, in response to the explosive growth of food safety problems, especially in developing countries. Biogenic amines are considered as important biomarker for monitoring food quality and assistant diagnosis of diseases ${ }^{1-3}$, since they usually are generated from the degradation of amino acids due to external microbial activity during food spoilage or endogenous tissue metabolisms ${ }^{4}$. At present, there are many methods for detecting biogenic amines, including liquid chromatography ${ }^{5}$, electrochemical devices $^{6}$, conductive polymer materials ${ }^{7}$, and optical sensors ${ }^{8}$. Among them, the optical sensors have attracted increasing attention, owing to high sensitivity and good selectivity ${ }^{9}$. However, the conventional optical sensors based on the change of the fluorescence intensity of only one luminogen always cannot perform well as expected, because the determination accuracy is easily influenced by the luminogen concentration, instrument and especially external environment, such as temperature, humidity, etc $^{10}$. Moreover, for one luminogen, there is only a minor change of fluorescence intensity in most cases, and the limited sensitivity of human eyes to the change of fluorescence brightness might increase the experimental errors, even cause a failure in the naked-eye detection mode ${ }^{11}$. Furthermore, it is noticeable that the common optical sensors are usually based on the small molecular probes in organic solvents ${ }^{12}$, thus they suffer from high toxicity and poor processibility. An ideal optical sensor in practice should exhibit not only excellent resolution and accuracy, but also simple operation, short analysis time, and easy visualization ${ }^{13}$.

The ratiometric fluorescent systems have shown great potential as ideal optical sensor, for which there always are two luminogens, generally one acts as the indicator of analyte and another as the internal reference ${ }^{14}$. The auto-calibration effect of the incorporated internal reference can significantly improve the detection accuracy based on the strong anti-interference ability ${ }^{15,16}$. More importantly, the sophisticated ratiometric systems established through the rational selection of different luminogens can precisely determine the analyte by the distinct fluorescent color change even with naked-eyes, i.e., visual detection or monitoring ${ }^{17}$. However, the difficulty in synthesis, poor processibility, high cost, and/or inferior responsiveness still restrict its scope of application in practice.

As the most abundant biopolymer on the earth, cellulose possesses many fascinating properties, such as biocompatibility, biodegradability, inexhaustible renew ability, and environmental friendliness ${ }^{18}$. Taking advantage of the reactive, numerous, and regularly arranged hydroxyl groups along cellulose chains, a wide variety of cellulose derivatives have been developed by simple reactions between functional substituents and active hydroxyls on the cellulose backbone ${ }^{19-21}$. They derive interesting properties from both cellulose and modified functional groups, including good solubility and processability, excellent flexibility, high mechanical strength, and so on. Recently, making full use of the structural characters and reactivity of cellulose skeleton, we successfully convert common aggregation-caused quenching (ACQ) luminogens into excellent solid fluorescent materials through covalently attaching the luminogens onto cellulose skeletons ${ }^{22}$. The synergy between the anchoring and diluting effect on luminogens of cellulose skeleton and the electrostatic repulsion among the ACQ luminogens efficiently inhibits the aggregation and the self-quenching of luminogens.

In this work, we design a cellulose-based ratiometric fluorescent material and propose a visualization method for monitoring the freshness of seafood. Fluorescein isothiocyanate (FITC, green, biogenic-amines indicator) and protoporphyrin IX (PpIX, red, internal reference) are covalently bonded onto cellulose acetate (CA) chains, respectively. Subsequently, the responsive ratiometric fluorescent materials are obtained by blending the cellulose derivatives CA-FITC and CA-PpIX together. The initial fluorescence color is finely tuned according to different blending ratios. The as-prepared ratiometric fluorescent materials not only exhibit fast and reversible response to biogenic amines, but also possess great processibility, thus they readily serve as a smart tag to provide practical applications in food industry. Compared with two standard methods for monitoring seafood freshness, including the total volatile basic nitrogen (TVBN) and the colony forming units (CFU), the resultant smart tag provides a high-contrast color change for real-time, visual, and accurate monitoring seafood freshness.

\section{Results}

Construction of the ratiometric fluorescent materials. Two common organic fluorophores FITC and PpIX were chosen, where FITC acted as an indicator, while PpIX as an internal reference. In general, due to the planar $\pi$-conjugation structures, they suffered from serious aggregation-caused fluorescence quenching (ACQ) phenomenon. Therefore, their emissions were utterly quenched in the solid state $^{23}$. In previous work, our group have developed a universal strategy to fabricate solid fluorescent material with high quantum yield by utilizing the anchoring and diluting effect of cellulose skeleton $22,24,25$. Based on this method, FITC and PpIX were covalently linked to cellulose acetate, respectively. As the images inserted in Fig. 1, green-emitting and red-emitting cellulosebased solid fluorescent materials are obtained.

Notably, the resultant green-emitting CA-FITC and redemitting CA-PpIX have the partially overlapped excitation bands, indicating that they can be excited at a same wavelength ranged from 350 to $525 \mathrm{~nm}$ (Supplementary Fig. 1). Moreover, they exhibit two distinct fluorescence colors according to their completely separated emission bands $(475-600 \mathrm{~nm}$ and 600-700 nm, respectively). Therefore, by simply mixing these two materials and adjusting their mix ratios, cellulose-based dualemission fluorescent materials with different initial fluorescence colors, from red to green, are obtained as seen in Fig. $1 \mathrm{~b}$.

${ }^{1} \mathrm{H}-\mathrm{NMR}$ and FTIR spectra of CA-FITC and CA-PpIX (Supplementary Fig. 2 and Supplementary Fig. 3) demonstrate that the two luminogens are successfully bonded on cellulose acetate, owing to the appearance of the characteristic peaks of FITC (6.4-6.8 ppm) and PpIX (6.2-6.7 ppm). However, the contents of luminogens on cellulose acetate are too low to be calculated by ${ }^{1} \mathrm{H}-\mathrm{NMR}$ spectra accurately. Thus, UV-vis spectroscopy was employed to determine the degree of substitution (DS) of luminogens, as shown in Supplementary Fig. 4 and Supplementary Fig. 5. The DS values of FITC and PpIX are 0.0114 and 0.0019 , respectively. Considering that the degree of polymerization of cellulose acetate is 112 , there is one FITC molecule per polymer chain and one PpIX molecule per every five polymer chains. Compared with physically blending the luminogens in polymer matrix ${ }^{26}$, chemical bonding is more efficient to achieve the homogeneous distribution at the molecular scale and relieve their aggregation behavior. Moreover, the chemical immobilizing effect of cellulose acetate could efficiently prevent the migration and leakage of the luminogens during usage, which is crucial to monitor foods.

Due to a negligible amount of luminogens are introduced onto cellulose acetate, the as-prepared materials still exhibit the excellent processibility of cellulose derivatives and can be readily transformed into various material forms ${ }^{27,28}$. As shown in Fig. 2, via a solution processing, the cellulose-based dual-emission fluorescent material $\left(m_{\mathrm{CA} \text {-FITC }} / m_{\mathrm{CA} \text {-P IX }}=1: 5\right)$ with a red initial fluorescence is fabricated into pattern printing, coating on plastic, flexible transparent film, and nanofibrous membrane by 
a

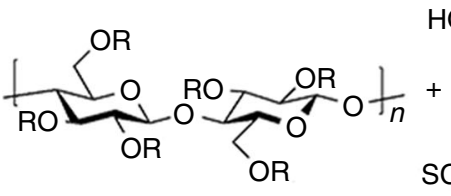

$\mathrm{R}=\mathrm{COCH}_{3}$ or $\mathrm{H}$<smiles></smiles>

DBTDL

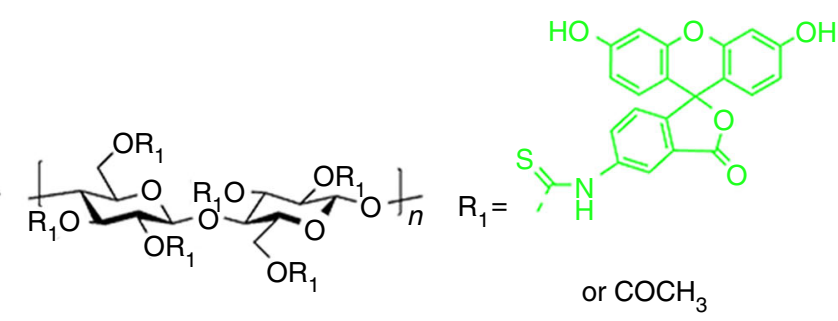

or $\mathrm{H}$

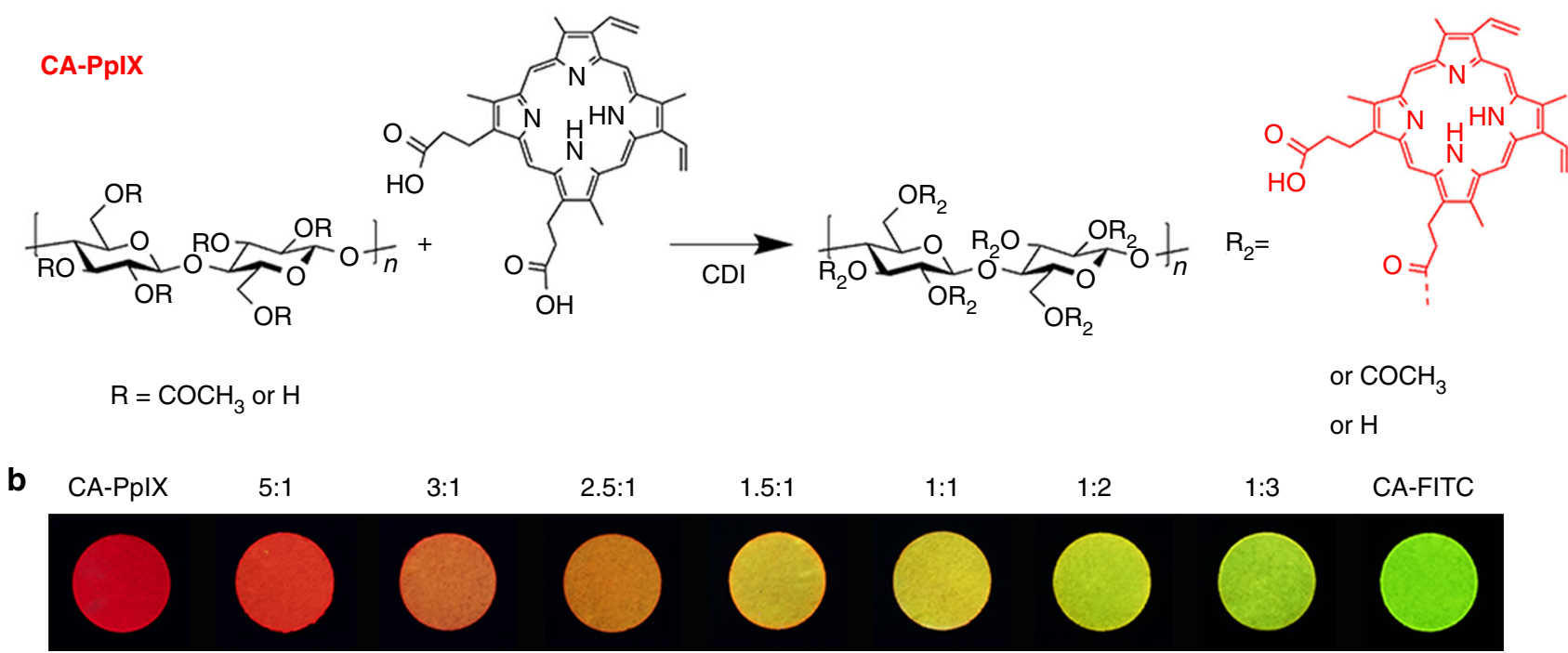

Fig. 1 Preparation of cellulose-based ratiometric fluorescent materials. a Synthetic routes of CA-FITC and CA-PpIX. b Cellulose-based dual-emission solid fluorescent materials with different mix ratio of CA-PpIX to CA-FITC (w/w), namely 1:0, 5:1, 3:1, 2.5:1, 1.5:1, 1:1, 1:2, 1:3, 0:1, respectively, under 365 nm UV light

a

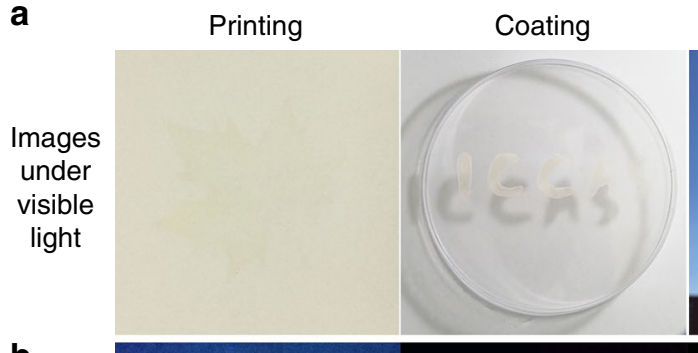

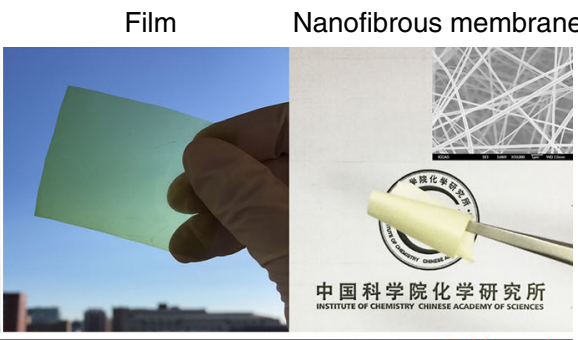

b

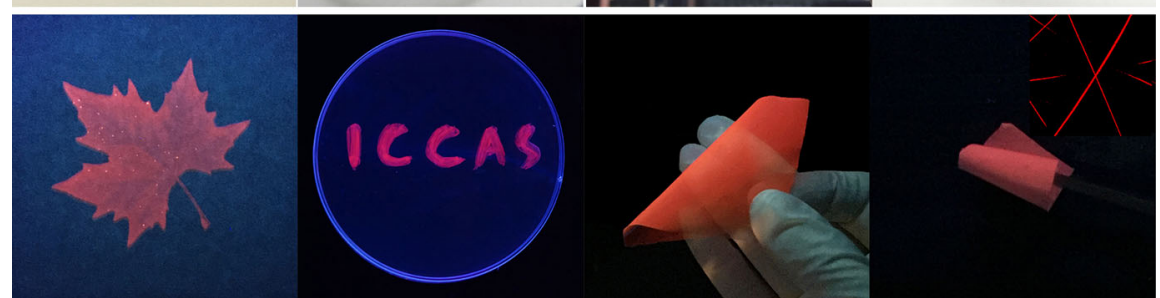

Fig. 2 Processability of cellulose-based ratiometric fluorescent materials. Images of a cellulose-based dual-emission fluorescent material with a red initial fluorescence in different material forms under a visible light and $\mathbf{b} 365 \mathrm{~nm}$ UV light. The insets are SEM and CLSM images of nanofibrous membrane, respectively

electrospinning. The images of scanning electron microscopy (SEM) and confocal laser scanning microscopy (CLSM) confirm that the smooth, uniform, and continous nanofibers are formed with a mean diameter of $200 \mathrm{~nm}$. The printing and coating technology make the as-prepared materials very suitable for mass production. Benefiting from the non-toxicity, biodegradability, low cost, and excellent mechanical property of cellulose acetate ${ }^{29,30}$, the obtained dual-emission fluorescent cellulose derivatives are expected to be used as intelligent packaging and smart tag.

Amine-response behavior. We used the dual-emission fluorescent cellulose materials as a ratiometric sensor for detecting 
ammonia, and the schematic illustration for the sensing mechanism is shown in Fig. 3a. When CA-FITC was blended with CA-PpIX in the ratio of 5:1, the obtained cellulose-based ratiometric fluorescent material exhibited a red fluorescence at the beginning. Upon exposing to ammonia, a proton of FITC was deprived and the molecular structure change resulted in the enhancement of green fluorescence ${ }^{31}$, which was confirmed by the change of ${ }^{1} \mathrm{H}$-NMR and UV-vis spectra of FITC (Supplementary Fig. 6). Protoporphyrin IX is irresponsive to ammonia, thus its red fluorescence remained unchanged. With the increase of the ammonia concentration, the green fluorescence of FITC started to be dominant. Thus, an obvious fluorescence color change occurred from red to orange, yellow or green, which was sufficiently clear to be recognized by any untrained observer.

The fluorescence spectra of the above ratiometric fluorescent material with a red initial fluorescence color were shown in Fig. 3b. They exhibits two characteristic emission bands of FITC at $525 \mathrm{~nm}$ and PpIX at $670 \mathrm{~nm}$. Upon exposing to ammonia, the fluorescence intensity of FITC increases with the concentration increase of ammonia, while that of PpIX remains constant. In the ammonia concentration range of $5.0 \mathrm{ppm}$ to $2.5 \times 10^{4} \mathrm{ppm}$, the fluorescence intensity ratio of CA-FITC to CA-PpIX $\left(I_{\mathrm{FITC}} / I_{\mathrm{PpIX}}\right)$ is linearly proportional to the logarithmic concentration of ammonia, as seen in Fig. 3c. Based on the calibration equation, we are able to detect ammonia quantitatively. Furthermore, the experiment results demonstrate that along with the increase of the concentration of ammonia, the fluorescence color simultaneously changes from red, to orange, to yellow and to green, which is consistent with our design. More importantly, the response to ammonia of the cellulose-based ratiometric fluorescent material is reversible. After a heating at $60^{\circ} \mathrm{C}$ or degassing under vacuum, the fluorescence of FITC quenches, and the fluorescence color returns to its original state, as shown in Fig. 3d. After four cycles, the material still maintains good ammonia responsiveness and high stability. Therefore, the prepared cellulose-based ratiometric fluorescent materials can be re-used for ammonia sensing. This ratiometric approach can effectively eliminate the interference of external environment and processing mode, thus, a more credible and reproducible result can be obtained. Moreover, if a droplet of the ammonia was added on the nanofibers membrane, a rapid response occurred, and the fluorescence color changed instantly $(<1 \mathrm{~s})$, as shown in Supplementary Movie 1. Such a sensitive, color change, linearly-dependent and rapid response to ammonia of the cellulose-based ratiometric fluorescent materials indicates that they can be used as a powerful tool to fast and accurately detect ammonia by naked-eyes in real time without the assistance of sophisticated instrument.

The above ratiometric fluorescent material exhibited a fast response to other organic amines also. As shown in Fig. 3e, the fluorescence intensity of the ratiometric fluorescent material gives a distinct change upon contacting with $\mathrm{NHEt}_{2}$, pyrrolidine, benzylamine, morpholine, putrescine, histamine, hydrazine, and $\mathrm{NEt}_{3}$, except aniline and urea. In addition, the change degrees of the fluorescence intensity induced by those organic amines are different with each other, because of their different basicity. The $I_{\mathrm{FITC}} / I_{\mathrm{PpIX}}$ of the control group is about 0.6 . At a concentration of amines of $2500 \mathrm{ppm}$, the $I_{\mathrm{FITC}} / I_{\mathrm{PpIX}}$ for $\mathrm{NH}_{3}$ increases to about 5.4, while those for $\mathrm{NHEt}_{2}$, pyrrolidine, benzylamine, morpholine, putrescine, histamine, hydrazine, and $\mathrm{NEt}_{3}$ are 4.9, 4.4, 4.3, $4.0,3.6,3.5,3.3$, and 1.8 , respectively. These organic amines, $\mathrm{NH}_{3}$, $\mathrm{NHEt}_{2}$, putrescine, histamine, and $\mathrm{NEt}_{3}$, are the important components of biogenic amines released by degradation of amino acids, thus, the ratiometric fluorescent materials can be used as an efficient indicator for monitoring metabolic process, such as food spoilage and some diseases occurrence $\mathrm{e}^{32,33}$
Monitoring of seafood freshness. Using shrimps as an example, we tested whether the prepared materials can be used as a smart tag for monitoring the seafood freshness. Red represents fresh, yellow represents slight spoilage, and green represents spoilage. The electrospun membranes consisted of nanofibers are employed for the subsequent test, owing to their large specific surface area and high porosity ${ }^{34}$. As shown in Fig. 4, a piece of electrospun membrane is fixed at the inside top of each packing box of the shrimp which was stored at three different temperatures for 1-5 days. After only one day storage at $25^{\circ} \mathrm{C}$ and $4{ }^{\circ} \mathrm{C}$, the fluorescence color of the membrane turned to reseda and yellow, respectively. After five days storage at $-16^{\circ} \mathrm{C}$, the fluorescence color of the membrane changed to yellow. As a control, electrospun membranes with wet tissues stored at different temperature for 5 days, and there was no change of the fluorescence color (Supplementary Fig. 7). Except the shrimp, the freshness of crab has been successfully and visually monitored by the smart tag (Supplementary Fig. 8).

In order to confirm the validity of the smart tag, we compared the results obtained from the smart tag with two standard reference methods, the total volatile basic nitrogen (TVBN) and the colony forming units (CFU), which are widely used by food inspection administration ${ }^{35-37}$. We have test the TVBN content in shrimps based on the standard method, as shown in Supplementary Fig. 9. The TVBN scales for raw shrimps are: $<12 \mathrm{mg} / 100 \mathrm{~g}$ for fresh, $12-20 \mathrm{mg} / 100 \mathrm{~g}$ for edible but slightly decomposed, $20-25 \mathrm{mg} / 100 \mathrm{~g}$ for borderline, and $>25 \mathrm{mg} / 100 \mathrm{~g}$ for inedible and spoiling ${ }^{35}$. The TVBN content in fresh shrimps is about $6.5 \mathrm{mg} / 100 \mathrm{~g}$. When the shrimps have been stored at $25^{\circ} \mathrm{C}$ for 1 day, the TVBN content sharply increases to $132 \mathrm{mg} / 100 \mathrm{~g}$, which means the shrimps have been totally spoiling. At the same time, the fluorescence color of the smart tag in the package changes from red to bright green, which also indicates the spoilage of the shrimps. When the shrimps have been stored at $4{ }^{\circ} \mathrm{C}$ for 1 day, the TVBN content increases to $14 \mathrm{mg} / 100 \mathrm{~g}$, and the smart tag changes its fluorescence color from red to light yellow. Both the phenomena claim that the shrimps are slightly decomposed. When the shrimps have been stored at $4{ }^{\circ} \mathrm{C}$ for $3-5$ days, the TVBN content is higher than $24 \mathrm{mg} / 100 \mathrm{~g}$, and the smart tag changes to green, which clearly demonstrates the spoilage of the shrimps. When the shrimps have been stored at $-16^{\circ} \mathrm{C}$ for 5 days, the TVBN content slightly increases to about $10 \mathrm{mg} /$ $100 \mathrm{~g}$, and the fluorescence color of the smart tag changes from red to light yellow, which means the shrimps are slightly decomposed. The TVBN content in crabs was tested too. As shown in Supplementary Fig. 10, the TVBN content in fresh carb is about $5.8 \mathrm{mg} / 100 \mathrm{~g}$. After storage at $25^{\circ} \mathrm{C}$ for 1 day, the TVBN content is about $15 \mathrm{mg} / 100 \mathrm{~g}$ and the smart tag changes to yellow. After storage at $25^{\circ} \mathrm{C}$ for 3 days, the TVBN content is about $104 \mathrm{mg} / 100 \mathrm{~g}$ and the smart tag changes to green, which represents the spoilage of the crabs. When the crabs have been stored at $4{ }^{\circ} \mathrm{C}$ for 1 day and 3 days, the TVBN contents increase to $8.1 \mathrm{mg} / 100 \mathrm{~g}$ and $21 \mathrm{mg} / 100 \mathrm{~g}$, respectively. The smart tag changes from red to orange (1 day) and yellow (3 days), which clearly demonstrates the slight decomposition of the crabs. When the crabs have been stored at $-16^{\circ} \mathrm{C}$ for 3 days, the TVBN content increases gradually to about $14 \mathrm{mg} / 100 \mathrm{~g}$, and the smart tag changes from red to yellow. These experiment results reveal that the color-change phenomena of the smart tag are completely consistent with the results of the standard TVBN test, so the freshness of the shrimps and crabs can be simply and accurately detected by the smart tag. Taking advantage of this property, the smart tags could be readily applied in the supply chain of shrimps and crabs for real-time and visual monitoring the freshness of shrimps and crabs. 


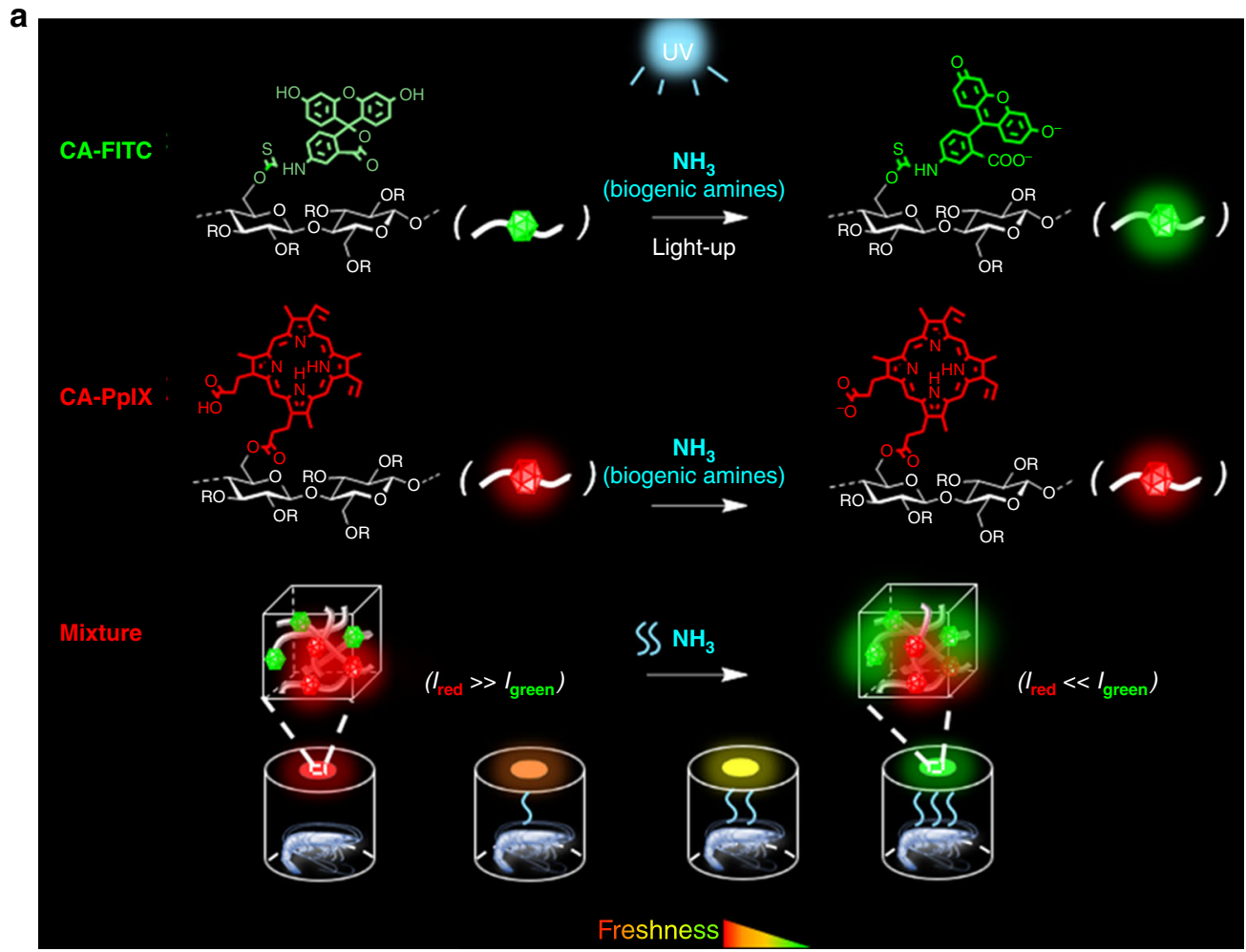

b

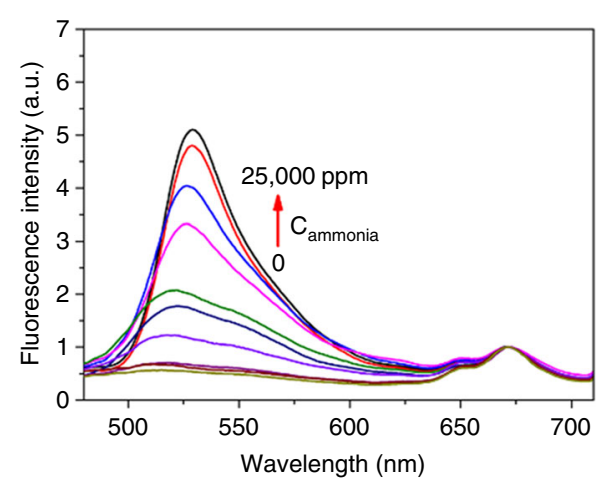

d

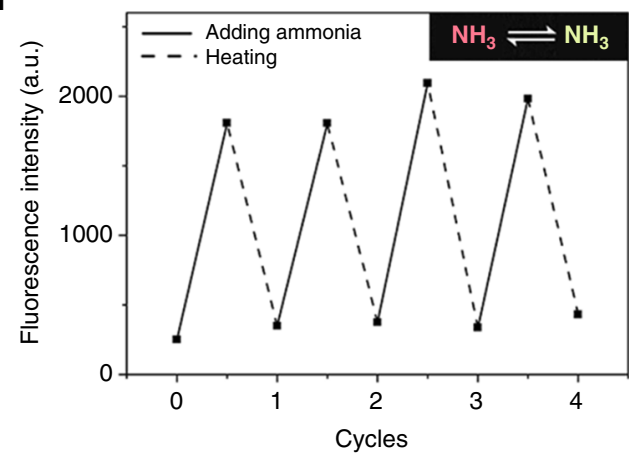

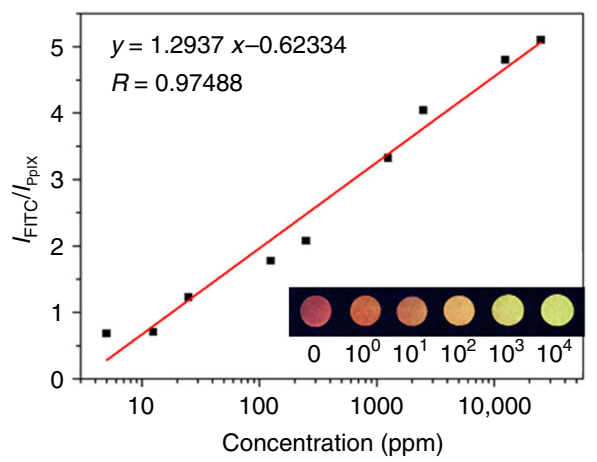

e

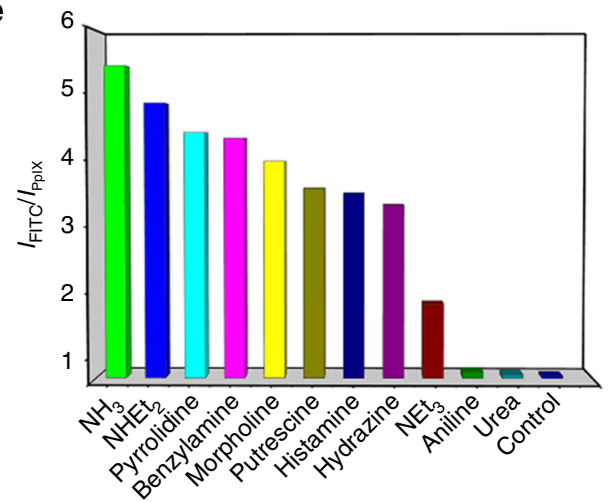

Fig. 3 Amine-response behavior of cellulose-based ratiometric fluorescent materials. a Schematic illustration for the mechanism of the cellulose-based ratiometric fluorescent material. $\mathbf{b}$ Emission spectra of the nanofibrous membrane dipping in different concentrations of ammonia. $\mathbf{c}$ Plot of the fluorescence intensity ratio of CA-FITC to CA-PpIX $\left(I_{\mathrm{FITC}} / I_{\mathrm{PpIX}}\right)$ versus concentration of ammonia. Inset: fluorescence images of the membrane treated with a series of different concentrations of ammonia. d Fluorescence enhancement and quenching cycles of the emission peak at $\lambda_{\mathrm{em}}=525 \mathrm{~nm}$ upon the nanofibrous membrane dipping in $2500 \mathrm{ppm}$ ammonia and following heated. Inset: reversible color change of the nanofibrous membrane in a cycle. e Fluorescence response of the nanofibrous membrane to ammonia and other organic amines in water ( 2500 ppm). Excitation wavelength, $365 \mathrm{~nm}$ 


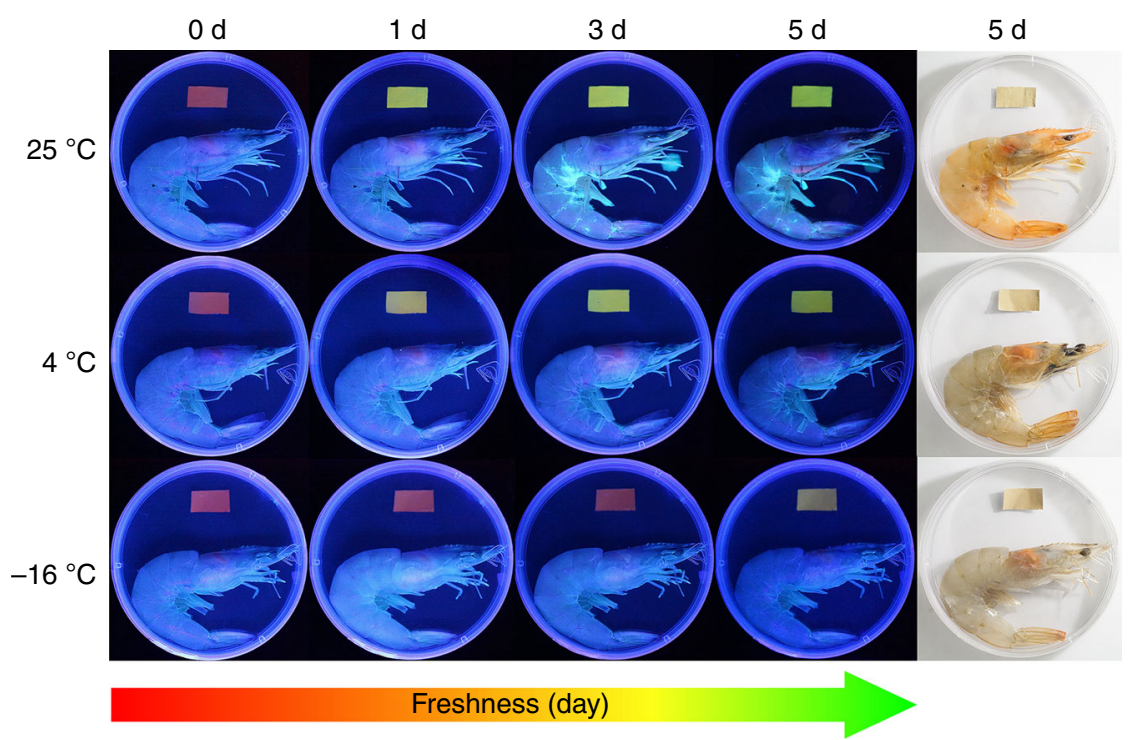

Fig. 4 Monitoring of shrimp freshness by the smart trademark. Images of the cellulose-based ratiometric fluorescent material with a red initial fluorescence as a smart trademark for monitoring the freshness of shrimps stored at different conditions

The colony forming units (CFU) in shrimps and crabs were also measured for comparison. Actually, it's hard to determine the CFU limit value which represents the total spoilage of shrimps or crabs, because original CFU of shrimps and crabs is subjected to various factors, such as species, producing area and fishing time. Therefore, there is no national standard about this issue. But, the change of $\mathrm{CFU}$ value during storage can be generally used to evaluate the freshness of shrimps and crabs. A significant increase of CFU means the decrease of freshness ${ }^{38,39}$. The trend of the CFU results is in accord with that of the TVBN results, as shown in Supplementary Fig. 11 and Supplementary Fig. 12. When the fresh shrimps have been stored at $25^{\circ} \mathrm{C}$ for 1 day, the $\mathrm{CFU}$ value grows exponentially, so the freshness decreases sharply ${ }^{40,41}$. When the shrimps have been stored at $4^{\circ} \mathrm{C}$, the CFU value decreases at first because of the death of the chillintolerable microorganism, then significantly increases, which means the freshness begin to decrease. When the shrimps have been stored at $-16^{\circ} \mathrm{C}$ for $1-7$ days, the $\mathrm{CFU}$ value also dramatically decreases at first, then increases slowly. The increase of bacteria means a decrease of the freshness. Comparing with the $\mathrm{CFU}$ value in the shrimps stored at $4{ }^{\circ} \mathrm{C}$, the $\mathrm{CFU}$ value at $-16^{\circ} \mathrm{C}$ remains at a lower level. Thus, the shrimps are just slight decomposed after 5 days. The change rule of CFU in crabs is similar with that of shrimps. When the crabs have been stored at $25^{\circ} \mathrm{C}$, the $\mathrm{CFU}$ value increases sharply, as a result, the freshness of crabs decreases. When the crabs have been stored at $4{ }^{\circ} \mathrm{C}$, the CFU decreases at first and increases after 3 days, so, after a 3-day storage, the freshness of crabs decreases. Therefore, the smart tags could be readily applied in the supply chain of shrimps and crabs for real-time and visual monitoring the freshness of shrimps and crabs.

\section{Discussion}

Overall, simple, fast, and accurate detection of food freshness has great significance to ensure food safety, which provides practical applications in food business. We developed a cellulose-based ratiometric fluorescent material with superior amine-response by covalently linking FITC and PpIX luminogens onto cellulose. Green-emitting CA-FITC served as a biogenic-amines indicator, while red-emitting CA-PpIX as an internal reference. The asprepared material exhibited a sensitive, color change, linearly dependent, reversible, and rapid response to biogenic amines, including $\mathrm{NH}_{3}$, pyrrolidine, benzylamine, morpholine, putrescine, histamine, hydrazine, and $\mathrm{NEt}_{3}$. Upon contacting with biogenic amines, particularly ammonia, their fluorescence color immediately changed from red to orange or green, and the fluorescence intensity ratio of CA-FITC to CA-PpIX $\left(I_{\mathrm{FITC}} / I_{\mathrm{PpIX}}\right)$ was linearly proportional to the logarithmic concentration of ammonia in a wide range of $5.0 \mathrm{ppm}$ to $2.5 \times 10^{4} \mathrm{ppm}$. Such a promising responsiveness offers a great opportunity to fast and accurately detect biogenic amines by naked-eyes in real time. Besides, taking advantages of the good solubility, processibility, and biocompatibility of cellulose derivatives, the ratiometric fluorescent material can be fabricated into many material forms, such as intelligent package and smart tag. The electrospun membrane was employed as a simple, low-cost, high-contrasting, quick-responsive, and smart trademark for visual monitoring the freshness of shrimp and crab successfully, which was validated against the standard food-monitoring methods, TVBN, and microbial activity. These materials have great significance to ensure food safety and provide practical applications in food business.

\section{Methods}

Materials. Cellulose acetate $(\mathrm{Mn}=30000, \mathrm{DS}=2.5)$ was used after drying in the vacuum oven at $70{ }^{\circ} \mathrm{C}$ for $24 \mathrm{~h}$ caisothiocyanate (FITC), protoporphyrin IX (PpIX), dibutyltindilaurate (DBTDL), $N, N^{\prime}$-carbonyldiimidazole (CDI) were purchased from J\& K Scientific Ltd. and used as received. N,N'-Dimethylformamide (DMF) was dehydrated by $\mathrm{CaH}_{2}$ and preserved with $3 \AA$ molecular sieve after distillation Super-dry dimethyl sulfoxide (DMSO) with molecular sieves was purchased from J\& K Scientific Ltd. and used as received. Ammonia $\left(\mathrm{NH}_{3}, 25 \%\right.$ in water), diethylamine $\left(\mathrm{NHEt}_{2}\right)$, triethylamine $\left(\mathrm{NEt}_{3}\right)$, and urea were purchased from Beijing Chemical Works and used as received. Aniline, benzylamine, hydrazine (64\%), and histamine were purchased from J\& K Scientific Ltd. and used as received. Mor pholine, pyrrolidine, and putrescine were purchased from Sigma-Aldrich, Inc. and used as received.

Synthesis of CA-FITC. The synthesis was performed in the Schlenk line. Firstly, 3 mmol cellulose acetate was dissolved in $20 \mathrm{~mL}$ of DMF at $80^{\circ} \mathrm{C}$ to get a homogeneous solution. Then, $0.1 \mathrm{mmol}$ FITC and $1.5 \mathrm{mmol}$ DBTDL as catalyst were added into the cellulose acetate solution followed by stirring at $100^{\circ} \mathrm{C}$ for $4 \mathrm{~h}$. The product was precipitated in the mixed solvent of methanol and water $(\mathrm{v} / \mathrm{v}=1 / 1)$ The generated precipitation was filtered and further washed three times followed by drying in the vacuum oven at $80^{\circ} \mathrm{C}$ for $24 \mathrm{~h}$ to offer a yellow powder.

Synthesis of CA-PpIX. Cellulose acetate $(4 \mathrm{mmol})$ was dissolved in $20 \mathrm{~mL}$ of DMSO. In another flask, $0.0667 \mathrm{mmol}$ PpIX was activated by CDI. Then, the prepared PpIX solution was added into the solution of cellulose acetate. The 
obtained mixture was stirred at $80^{\circ} \mathrm{C}$ for $20 \mathrm{~h}$. The final product was precipitated in ethanol and further washed three times followed by drying in the vacuum oven at $80^{\circ} \mathrm{C}$ for $24 \mathrm{~h}$ to offer a brown powder.

Preparation of ratiometric fluorescent materials. A homogeneous solution was prepared by dissolving CA-FITC and CA-PpIX in DMF with a mass ratio of 1:3. By using EPSON ME-10 printer and replacing the printing ink with the solution of CA-FITC and CA-PpIX, a designed pattern was obtained. Coating on plastic was performed by writing with Chinese brush. The transparent film was prepared by casting the solution on the glass and volatilizing the solvent. The nanofibrous membrane was fabricated by electrospinning. Firstly, a solution was prepared by dissolving CA-FITC and CA-PpIX in DMAc/acetone $(\mathrm{v} / \mathrm{v}=1 / 2)$ at the concentration of $15 \mathrm{wt} \%$. Then, the solution in the syringe was electrospun at a voltage of $20 \mathrm{kV}$ with a tip-to-collector distance of $15 \mathrm{~cm}$ and a flow rate of $0.6 \mathrm{~mL} / \mathrm{h}$ to afford a nanofibrous membrane. DW-P303-0.1 AC high voltage power supply was used as the power source, and LSP01-1A from LongerPump was used as the injection pump.

Preparation and detection of amine solutions. Commercial ammonia was diluted with different quantity of deionized water to obtain a series of ammonia solutions with known concentration. Then, the nanofibrous membrane was immersed in the ammonia solution for $3 \mathrm{~min}$. After being taken out from the solution, the fluorescence of the nanofibrous membrane was recorded as soon as possible. The preparation and detection process of other amines solutions were the same as that of ammonia.

Detection of the total volatile basic nitrogen. According to the Chinese Standard GB 5009.228-2016, the total volatile basic nitrogen (TVBN) in shrimps and crabs was determined. All the shrimps were removed the shell and head. The crabs were removed the shell. The meat of shrimp and carb was used for the experiments. All experiments were performed in triplicate.

Detection of microbial count in shrimps and crabs. According to the Chinese Standard GB 4789.2-2010 for food safety, the colony forming units (CFU) in shrimps and crabs were determined. Each sample was derived from three shrimps or three crabs in the same storage environment and was given an alcohol sterilization before detection. The final experiment results were obtained by plate counting method.

Characterization. ${ }^{1} \mathrm{H}$-NMR spectrum was measured on Bruker Avance $400 \mathrm{M}$ NMR spectrometer. Lambda 35 spectrophotometer from Perkin-Elmer was used to record UV-vis absorption. Fluorescence emission and excitation spectra were measured on HITACHI F-4500 fluorescence spectrometer. The morphology of the nanofibrous membrane was investigated by SEM (JSM6700F) and CLSM (OLYMPUS, FV1000-IX81). Thermogravimetric analyzer TGA 8000 from Perkin-Elmer was used to record the TGA curves of CA-FITC and CA-PpIX under nitrogen atmosphere. The FTIR spectra were measured by NICOLET 6700 from Thermo-Fisher Scientific. Differential scanning calorimetry (DSC) was conducted on TA-Q200 differential scanning calorimeter (TA Instruments, USA) under nitrogen atmosphere. The total volatile basic nitrogen (TVBN) was measured by K-375 Kjeldahl apparatus from BUCHI Corporation.

\section{Data availability}

All relevant data are included in this Article and its Supplementary Information files. The freshness detection data have been submitted to https://figshare.com/. https://doi.org/ 10.6084/m9.figshare.7545638.

Received: 5 January 2018 Accepted: 18 January 2019

Published online: 15 February 2019

\section{References}

1. Vinci, G. \& Antonelli, M. L. Biogenic amines: uality index of freshness in red and white meat. Food Control. 13, 519-524 (2002).

2. Pacquit, A. et al. Development of a smart packaging for the monitoring of fish spoilage. Food Chem. 102, 466-470 (2007).

3. Shang, C. D. et al. A high performance fluorescent arylamine sensor toward lung cancer sniffing. Sens. Actuators, B 241, 1316-1323 (2017).

4. Silla, S. M. H. Biogenic amines: their importance in foods. Int. J. Food Microbiol. 29, 213-231 (1996).

5. Onal, A., Tekkeli, S. E. K. \& Onal, C. A review of the liquid chromatographic methods for the determination of biogenic amines in foods. Food Chem. 138, 509-515 (2013).
6. Zaczek, R. \& Coyle, J. T. Rapid and simple method for measuring biogenic amines and metabolites in brain homogenates by HPLC-electrochemical detection. J. Neural Transm. 53, 1-5 (1982).

7. Kumara, L., Rawalb, I., Kaurb, A. \& Annapoornib, S. Flexible room temperature ammonia sensor based on polyaniline. Sens. Actuators, B 240, 408-416 (2017).

8. Gao, M. et al. Fluorescent light-up detection of amine vapors based on aggregation-induced emission. ACS Sens. 1, 179-184 (2016).

9. Lippert, A. R., New, E. J. \& Chang, C. J. Reaction-based fluorescent probes for selective imaging of hydrogen sulfide in living cells. J. Am. Chem. Soc. 133, 10078-10080 (2011).

10. Liu, X. J., Zhang, N., Bing, T. \& Shangguan, D. H. Carbon dots based dualemission silica nanoparticles as a ratiometric nanosensor for $\mathrm{Cu}^{2+}$. Anal. Chem. 86, 2289-2296 (2014).

11. Chow, C. F., Lam, M. H. W. \& Wong, W. Y. Design and synthesis of heterobimetallic $\mathrm{Ru}(\mathrm{II})-\mathrm{Ln}(\mathrm{III})$ complexes as chemodosimetric ensembles for the detection of biogenic amine odorants. Anal. Chem. 85, 8246-8253 (2013).

12. Han, T. Y. et al. A fluorescence-switchable luminogen in the solid state: a sensitive and selective sensor for the fast "turn-on" detection of primary amine gas. Chem. Commun. 49, 4848-4850 (2013).

13. Kuswandi, B. et al. Smart packaging: Sensors for monitoring of food quality and safety. Sens. Instrumen. Food Qual. 5, 137-146 (2011).

14. Wang, M. L. et al. FITC-modified PPy nanotubes embedded in nanoporous AAO membrane can detect trace PCB20 via fluorescence ratiometric measurement. Chem. Commun. 47, 3808-3810 (2011).

15. Chen, Y. C. et al. A ratiometric fluorescent probe for rapid detection of hydrogen sulfide in mitochondria. Angew. Chem. Int. Ed. 125, 1732-1735 (2013).

16. Wang, R. F. et al. A hydrogen-bonded-supramolecular-polymer-based nanoprobe for ratiometric oxygen sensing in living cells. Adv. Funct. Mater 26, 5419-5425 (2016)

17. Hao, T. F. et al. Surface modification and ratiometric fluorescence dual function enhancement for visual and fluorescent detection of glucose based on dual-emission quantum dots hybrid. Sens. Actuators, B 230, 70-76 (2016).

18. Klemm, D., Heublein, B., Fink, H. P. \& Bohn, A. Cellulose: fascinating biopolymer and sustainable raw material. Angew. Chem. Int. Ed. 44, 3358-3393 (2005)

19. Zhang, J. M. et al. Application of ionic liquids for dissolving cellulose and fabricating cellulose-based materials: state of the art and future trends. Mater. Chem. Front. 1, 1273-1290 (2017).

20. Seoud, O. A. E., Nawaz, H. \& Arêas, E. P. G. Chemistry and applications of polysaccharide solutions in strong electrolytes/dipolar aprotic solvents: an overview. Molecules 18, 1270-1313 (2013).

21. Rose, M. \& Palkovits, R. Cellulose-based sustainable polymers: state of the art and future trends. Macromol. Rapid Commun. 32, 1299-1311 (2011).

22. Tian, W. G. et al. Cellulose-based solid fluorescent materials. Adv. Opt. Mater. 4, 2044-2050 (2016).

23. Yuan, W. Z. et al. Changing the behavior of chromophores from aggregationcaused quenching to aggregation-induced emission: Development of highly efficient light emitters in the solid state. Adv. Mater. 22, 2159-2163 (2010).

24. Tian, W. G. et al. Phototunable full-color emission of cellulose-based dynamic fluorescent materials. Adv. Funct. Mater. 28, 1703548 (2018).

25. Nawaz, H. et al. Cellulose-based sensor containing phenanthroline for the highly-selective and rapid detection of $\mathrm{Fe}^{2+}$ ions with naked-eye and fluorescent dual-modes. ACS Appl. Mater. Interfaces 10, 2114-2121 (2018).

26. Preininger, C., Mohr, G. J., Klimant, I. \& Wolfbeis, O. S. Ammonia fluorosensors based on reversible lactonization of polymer-entrapped rhodamine dyes, and the effects of plasticizers. Anal. Chim. Acta 334, 113-123 (1996).

27. Mi, Q. Y., Ma, S. R., Yu, J., He, J. S. \& Zhang, J. Flexible and transparent cellulose aerogels with uniform nanoporous structure by a controlled regeneration process. ACS Sustain. Chem. Eng. 4, 656-660 (2016).

28. Zhang, J. J. et al. Renewable and superior thermal-resistant cellulose-based composite nonwoven as lithium-ion battery separator. ACS Appl. Mater. Interfaces 5, 128-134 (2013).

29. Yam, K. L., Takhistov, P. T. \& Miltz, J. Intelligent packaging: concepts and applications. J. Food Sci. 70, R1-R10 (2005).

30. Abad, E. et al. RFID smart tag for traceability and cold chain monitoring of goods: demonstration in an intercontinental fresh fish logistic chain. J. Food Eng. 93, 394-399 (2009).

31. Widmer, S. et al. Coumarin meets fluorescein: a Förster resonance energy transfer enhanced optical ammonia gas sensor. Analyst 139, 4335-4342 (2014).

32. Ghaly, A. E., Dave, D., Budge, S. \& Brooks, M. S. Fish spoilage mechanisms and preservation techniques: review. Am. J. Appl. Sci. 7, 859-877 (2010).

33. Broza, Y. Y. \& Haick, H. Nanomaterial-based sensors for detection of disease by volatile organic compounds. Nanomedicine 8, 785-806 (2013).

34. Zhao, Y. L. et al. Dendrimer-functionalized electrospun cellulose acetate nanofibers for targeted cancer cell capture applications. J. Mater. Chem. B 2, 7384-7393 (2014). 
35. Okpala, C. O. R., Choo, W. S. \& Dykes, G. A. Quality and shelf life assessment of Pacific white shrimp (Litopenaeus vannamei) freshly harvested and stored on ice. LWT - Food Sci. Technol. 55, 110-116 (2014).

36. Bazemore, R., Fu, S. G., Yoon, Y. \& Marshall, D. Major causes of shrimp spoilage and methods for assessment. ACS Symposium, 848, 223-234 (2003).

37. National Standard of the People's Republic of China. National Food Safety Standards for Fresh and Frozen Aquatic Animal (National Health and Family Planning Commission, 2015).

38. Nirmal, N. P. \& Benjakul, S. Effect of catechin and ferulic acid on melanosis and quality of Pacific white shrimp subjected to prior freeze-thawing during refrigerated storage. Food Control. 21, 1263-1271 (2010).

39. Zeng, Q. Z., Thorarinsdottir, K. A. \& Olafsdottir, G. Quality changes of shrimp (Pandalus borealis) stored under different cooling conditions. J. Food Sci. 70, S459-S466 (2005).

40. Pacquit, A. et al. Development of a volatile amine sensor for the monitoring of fish spoilage. Talanta 69, 515-520 (2006).

41. Anacleto, P. et al. Shelf-life of cooked edible crab (Cancer pagurus) stored under refrigerated Conditions. LWT-Food Sci. Technol. 44, 1376-1382 (2011).

\section{Acknowledgements}

This work was supported by the National Science Foundation of China (Nos. 51425307, 51573196, 21533012, and 91527306), the Program of Taishan Industry Leading Talents (Shandong Province), and the Youth Innovation Promotion Association CAS (No. 2018040).

\section{Author contribution}

R.J., J.Z., S.W., and J.Z. conceived the idea. R.J. performed the experiments. W.T. and H. B. offered help to R.J. for the experiments. R.J., W.T., J.Z., S.W., and J.Z. discussed the results and wrote the manuscript.

\section{Additional information}

Supplementary Information accompanies this paper at https://doi.org/10.1038/s41467019-08675-3.

Competing interests: The authors declare no competing interests.

Reprints and permission information is available online at http://npg.nature.com/ reprintsandpermissions/

Journal peer review information: Nature Communications thanks the anonymous reviewers for their contribution to the peer review of this work. Peer reviewer reports are available.

Publisher's note: Springer Nature remains neutral with regard to jurisdictional claims in published maps and institutional affiliations.

(c) (i) Open Access This article is licensed under a Creative Commons Attribution 4.0 International License, which permits use, sharing, adaptation, distribution and reproduction in any medium or format, as long as you give appropriate credit to the original author(s) and the source, provide a link to the Creative Commons license, and indicate if changes were made. The images or other third party material in this article are included in the article's Creative Commons license, unless indicated otherwise in a credit line to the material. If material is not included in the article's Creative Commons license and your intended use is not permitted by statutory regulation or exceeds the permitted use, you will need to obtain permission directly from the copyright holder. To view a copy of this license, visit http://creativecommons.org/ licenses/by/4.0/.

(C) The Author(s) 2019 